\title{
GENERALIZED SKEW POLYNOMIAL RINGS ${ }^{1}$
}

\author{
BY
}

JOHN DAUNS

\begin{abstract}
For a totally ordered cancellative semigroup $\Gamma$, a skew field $K$, let $K[\Gamma ; \phi]$ be a skew semigroup ring. If $x \in \Gamma, k \in K$, then $k x=x k^{x}$, where $k \rightarrow k^{x}$ is an endomorphism of $K$ depending on $x$. Ideals of $K[\Gamma ; \phi]$ are investigated for various semigroups or groups $\Gamma$.
\end{abstract}

Suppose that $\Gamma$ is a totally ordered cancellative semigroup $\Gamma \leqslant e=1$ whose principal right ideals form a chain. For a skew field $K$, let $\phi: \Gamma \rightarrow$ End $K$ be a semigroup homomorphism, where for each $x \in \Gamma, x \phi: K \rightarrow K, k \rightarrow k(x \phi)=k^{x} \in K$ is a monic ring endomorphism of $K$. Let $K[\Gamma ; \phi]$ be the skew semigroup ring where $k x=x k^{x}$.

Various descriptions of biprincipal (= both principal left and principal right) ideals as well as ideals of $K[\Gamma ; \phi]$ are obtained.

THEOREM. Under three additional assumptions on $\Gamma$, any biprincipal ideal $J \triangleleft K[\Gamma ; \phi]$ is uniquely of the form $J=s K[\Gamma ; \phi]=K[\Gamma ; \phi] s$, where $s \in \Gamma$ with $s \Gamma=\Gamma s$ and $s \phi \in$ Aut $K$.

THEOREM. Let $\Lambda$ be any totally ordered group and $\theta: \Lambda \rightarrow$ Aut $K \subset$ End $K$ a group homomorphism. Define $\Gamma=\{g \in \Lambda \mid g \leqslant e=1\}, R \equiv K[\Gamma ; \theta] \subset S \equiv K[\Lambda ; \theta]$. If I $\triangleleft S$ is biprincipal, then $I=\gamma S=S \gamma$ uniquely for some $\gamma \in R$ with $\gamma(e)=1$ and $\gamma R=R \gamma$.

Corollary. If in addition for any $e \neq y \in \Gamma, y \theta \in$ Aut $K$ is NOT inner, then $K[\Lambda ; \theta]$ is a simple ring.

Introduction. If $K$ is a skew field and $\psi$ is an endomorphism of $K$, then the ring $K[x ; \psi]$ of polynomials with right side coefficients in $K$ is called the skew polynomial ring, where $k x=x(k \psi)$ for $k \in K$. This ring is the very special case of a skew semigroup ring over the totally ordered infinite cyclic semigroup $\Gamma=\left\{\cdots<x^{2}<x\right.$ $<1\}$. In view of the general interest and many applications of skew polynomial rings - only a few of which may be found in [1, 2 and 16],-it seems surprising that only a few attempts have been made to generalize this familiar class of rings by

Received by the editors May 21, 1980.

1980 Mathematics Subject Classification. Primary 16A02, 16A05; Secondary 16A86, 16A39.

Key words and phrases. Totally ordered group and semigroup, skew polynomial ring, skew group ring, group power series division ring, valuation, simple ring.

'Parts of this paper have been presented-without any proofs-at the University of Illinois Carbondale Algebra Conference, April 1980. 
replacing the infinite cyclic semigroup by more general totally ordered cancellative semigroups $\Gamma$. The appropriate generalization and object of study of this paper is a skew semigroup ring $K[\Gamma ; \phi]$ where each semigroup element $x$ acts as an endomorphism $x \phi: K \rightarrow K, k \rightarrow k(x \phi)$ of $K$, and where $k x=x(k(x \phi)), k \in K$.

The type of ring $K[\Gamma ; \phi]$ studied here is of possible interest from two other points of view. First of all, skew group rings have only begun to be seriously studied recently [18], and any kind of a complete theory like [19] for them is still lacking. A skew semigroup ring is a very obvious generalization of this latter class of rings. Secondly, ordinary semigroup or group rings $K[\Gamma]$ arise naturally in the study of partially ordered rings as subrings of generalized power series rings [22, 5 and 4]. For example, with some additional assumptions, every lattice ordered field $F$ is a subfield of a power series field over the reals, where the usual integer exponents are replaced by elements of a lattice ordered abelian group. In this embedding $F$ contains the group subring of the power series field [4]. Moreover, ordinary semigroup and group rings over partially ordered groups and semigroups are not only studied for their own sake [5, 20, 7 and 9], but are also used as examples and counterexamples [14, 6]. In addition, they are useful for constructing division rings [12, p. 197; 17, p. 1499; 8; and 11].

An element $\gamma$ of a ring $R$ is called invariant if $\gamma R=R \gamma$. Here two sided ideals of the latter kind are studied in skew semigroup rings (Propositions 2.4, 3.1 and Theorem I). This leads to the construction of some simple rings (Theorem II and 3.6). As an immediate corollary, a complete description of the ideal structure of skew polynomial rings $K[x ; \theta] \subset K\left[x, x^{-1} ; \theta\right]$ is given (4.2). The present description here in 4.2 differs from $[15$, p. 38$]$ in that here it is shown that ideals of the above rings arise from central elements of $K[x ; \theta]$, while in [15, p. 38] central elements are not mentioned.

\section{Basics.}

1.1. Notation. For a skew field $K$, the set of all nonzero ring endomorphisms of $K$ form a multiplicative semigroup which here will be denoted by End $K$. Every element of End $K$ is monic and identity preserving. Elements of End $K$ will act on the right side of $K$, and End $K$ is right cancellative $(\sigma \rho=\tau \rho \Rightarrow \sigma=\tau)$.

Let $\Omega$ be a cancellative multiplicative semigroup with identity $e=1 \in \Omega$. Let $\phi$ : $\Omega \rightarrow$ End $K$ be a semigroup homomorphism. It is a consequence of the right cancellativity of $\Omega$ that $\phi$ preserves the identity, $e \phi=1 \in$ End $K$. For any $x, y \in \Omega$, the maps $x \phi, y \phi,(x y) \phi,(x \phi)(y \phi): K \rightarrow K$ are monic identity preserving ring homomorphisms, where $1(x \phi)=1$, and $(x y) \phi=x \phi(y \phi)$. For any $k, c \in K, k^{x}$ and $k^{-x}$ are defined below, and the following hold:

$$
\begin{gathered}
k^{x} \equiv k(x \phi)=k^{x \phi}, \quad k((x y) \phi)=\left(k^{x}\right)^{y}, \quad k^{e}=k^{e \phi}=k ; \\
k^{-x} \equiv 1 / k^{x}, \quad k^{-x}=(1 / k)^{x}=\left(k^{-1}\right)^{x}=\left(k^{x}\right)^{-1} . \\
(k-c)^{x}=k^{x}-c^{x}, \quad(k c)^{x}=k^{x} c^{x} .
\end{gathered}
$$

The automorphisms of $K$ form a group Aut $K$. If for some $z \in \Omega, z \phi \in$ Aut $K$ is an automorphism, and $(z \phi)^{-1} \in$ Aut $K$ is its inverse, then define $k^{1 / z}$ to be $k^{1 / z}=$ $k(z \phi)^{-1}$. In general, $1 / z \notin \Omega$ and $(z \phi)^{-1} \notin \Omega \phi ;$ also $k^{1 / z} \neq k^{-z}$. 
The support of any function $\alpha: \Omega \rightarrow K$ is the set supp $\alpha=\{x \in \Omega \mid \alpha(x) \neq 0\}$. The set of all such functions $\alpha, \beta: \Omega \rightarrow K$ with finite supports, $|\operatorname{supp} \alpha|<\infty$ and $|\operatorname{supp} \beta|<\infty$, under pointwise addition form an abelian group. It becomes an associative ring called the skew semigroup ring $K[\Omega ; \phi]$ with the product $\alpha \beta: \Omega \rightarrow K$ whose value at $z \in \Omega$ is

$$
\begin{array}{r}
(\alpha \beta)(z)=\sum\left\{\alpha(x)^{y} \beta(y) \mid(x, y) \in \Omega \times \Omega, x y=z\right\} \in K ; \\
\alpha(x), \alpha(x)^{y}, \beta(y) \in K .
\end{array}
$$

Alternatively and more frequently, elements $\alpha$ and $\beta$ of $K[\Omega ; \phi]$ are viewed as finite right $K$-linear combinations of elements of $\Omega$, in which case for any $z \in \Omega$ and $k \in K, k z=z k^{z}$, and hence $\alpha=\sum x \alpha(x), \beta=\sum y \beta(y), \alpha \beta=\sum x y \alpha(x)^{y} \beta(y)$. With this interpretation it becomes clear that $\Omega \subset K[\Omega ; \phi]$ is a multiplicative subsemigroup, and that $1=e \in \Omega$ is the identity element of this ring. The product $\alpha x$ : $\Omega \rightarrow K$ of the two ring elements $\alpha$ and $x$ should not be confused with the $x$-coefficient $\alpha(x) \in K$ of $\alpha$. It will always be clear from the context which of these two is used.

If every element $x \in \Omega$ acts as the identity $x \phi=1$ on $K$ then $\phi=1$ and $K[\Omega ; \phi]=K[\Omega ; 1]=K[\Omega]$ is the ordinary semigroup ring. When every semigroup element acts as an automorphism of $K$ then the image of $\phi$ is in Aut $K$. In this case $\phi$ will be replaced with $\theta$, where $\theta: \Omega \rightarrow$ Aut $K$. The same two symbols $\phi$ and $\theta$ will be used with the above meanings while the semigroup $\Omega$ will be replaced with various more specialized semigroups or groups.

In any ring $R$, the notation $J \triangleleft R$ means that $J$ is an ideal. An ideal $J \triangleleft R$ is called biprincipal if $J$ is of the form $J=\alpha R=R \beta$ for some $\alpha, \beta \in R$.

Three subsemigroups of $\Omega$ are defined below in 1.2 and 1.3 which will be useful in skew semigroup rings.

1.2. Subsemigroups. The center of any semigroup $\Omega$ will be denoted by $\Sigma(\Omega) \equiv$ center $\Omega$, and abbreviated as $\Sigma=\Sigma(\Omega)$ if $\Omega$ is fixed or understood.

If $\phi: \Omega \rightarrow$ End $K$ is as above, then all the elements of any subsemigroup of $\Omega$ or of all of $\Omega$ which induce inner automorphism of $K$ form a subsemigroup $\Omega_{\text {INN }}$. Thus an element $z \in \Omega$ belongs to $z \in \Omega_{\mathrm{INN}}$ if and only if there exists a $0 \neq d \in K^{*}=K \backslash\{0\}$ such that $k^{z}=d^{-1} k d$, or $k z d^{-1}=z d^{-1} k$ for all $k \in K$.

1.3. Invariant elements. For any cancellative semigroup such as $\Omega$, define the subsemigroup $\Omega_{\mathrm{IV}} \subseteq \Omega$ of the so-called invariant elements by $\Omega_{\mathrm{IV}}=\{s \in \Omega \mid s \Omega=$ $\Omega s\}$. For any ring $R$ define $R^{*}=F \backslash\{0\}$ and $R_{\mathrm{IV}}=\{\gamma \in R \mid \gamma$ is not a zero divisor, and $\gamma R=R \gamma\}$. If $R$ is a domain, then $R^{*}$ will be viewed as a multiplicative semigroup and thus $R_{\mathrm{IV}}=R_{\mathrm{IV}}^{*}$.

1.4. Totally ordered semigroups. A linearly or totally ordered set $\Gamma$ which is also a semigroup is a totally ordered semigroup if for any $x \leqslant y, z \in \Gamma$, also $z x \leqslant z y$ and $x z \leqslant y z$. Throughout $\Gamma$ will automatically denote a totally ordered cancellative multiplicative semigroup with identity $e=1 \in \Gamma(a<b, c \in \Gamma \Rightarrow c a<c b$, and $a c<b c$ ). A totally ordered group is a totally ordered semigroup which algebraically is a group. Throughout the symbol $\Lambda$ will denote a totally ordered group. For any subsets $A, B \subseteq \Gamma$ the notation $A \leqslant B$ will mean that $a \leqslant b$ for every $a \in A$ and 
$b \in B$. If $\Gamma$ is right Ore, then its right Ore quotient group $\Gamma \Gamma^{-1}=\left\{a b^{-1}=a / b \mid a, b\right.$ $\in \Gamma\}$ is a totally ordered group, where $a / b<c / d$ if as $<c t$ for any $s, t \in \Gamma$ such that $b s=d t$ (see [3, p. 52, Theorem 2-1], also [13, p. 168, Corollary 5], and [12, p. 161, Corollary 5]). Left Ore and $\Gamma^{-1} \Gamma$ are defined similarly. When $\Gamma$ is both left and right Ore, then $\Gamma \Gamma^{-1}=\Gamma^{-1} \Gamma$ both algebraically and as totally ordered groups.

For a skew field $K$ and a right Ore semigroup $\Gamma$, and for $\theta: \Gamma \rightarrow$ Aut $K$ as in 1.1, there is a unique extension of $\theta$ to a group homomorphism $\theta: \Gamma \Gamma^{-1} \rightarrow$ Aut $K$ which is also denoted by $\theta$.

Thus the symbol " $K[\Gamma ; \phi]$ " from now on means that $\Gamma$ is a totally ordered cancellative semigroup with $1=e \in \Gamma$, that $K$ is a skew field, and that $\phi: \Gamma \rightarrow$ End $K$. If, in addition, $\Gamma$ is right Ore and $\phi=\theta$, then $K[\Gamma ; \theta] \subset K\left[\Gamma \Gamma^{-1} ; \theta\right]$. It is a consequence of the total order on $\Gamma$ that all three of these rings are domains.

1.5. Subfields. For $\phi: \Omega \rightarrow$ End $K$ or $\phi: \Gamma \rightarrow$ End $K$, define $K_{\phi} \subseteq K$ to be the skew subfield of $K$ which is left elementwise fixed by $\Omega, K_{\phi}=\left\{k \in K \mid k^{2}=k\right.$ for all $z \in \Omega\}$. Let $C_{\phi} \subseteq$ center $K$ be the subfield $C_{\phi}=K_{\phi} \cap$ center $K$ of the center of $K$ which is left pointwise fixed by every element of $\Omega \phi \subseteq$ End $K$.

1.6. Suppose that the totally ordered cancellative semigroup $\Gamma$ also satisfies (a) $\Gamma \leqslant e=1$. Then

(i) the principal right ideals of $\Gamma$ form a chain if and only if, for any $x<y \in \Gamma$, it follows that $x \in y \Gamma$.

Now, in addition to (a) assume that (b) both the principal right and principal left ideals of $\Gamma$ are chains. For any subset of $\Gamma \Gamma^{-1}$ such as $\Gamma$, define $\Gamma^{-1}=\{1 / x \mid x \in \Gamma\}$ $\subset \Gamma \Gamma^{-1}$. Set $\Sigma=$ center $\Gamma$. Then $\Gamma^{-1}, \Sigma^{-1}, \Gamma_{\mathrm{INN}}^{-1}$, and $\Sigma_{\mathrm{INN}}^{-1}$ are subsemigroups of $\Gamma \Gamma^{-1}$ and

(ii) $\Gamma \Gamma^{-1}=\Gamma \cup \Gamma^{-1}, \Gamma \cap \Gamma^{-1}=\{e\}$,

(iii) center $\Gamma \Gamma^{-1}=\Sigma \cup \Sigma^{-1}$,

(iv) $\Gamma_{\text {INN }}$ satisfies hypotheses (a) and (b), and $\left(\Gamma \Gamma^{-1}\right)_{\text {INN }}=\Gamma_{\text {INN }} \Gamma_{\text {INN }}^{-1}=\Gamma_{\text {INN }} \cup$ $\Gamma_{\text {INN }}^{-1}$; in particular

(v) $\left(\Gamma \Gamma^{-1}\right)_{\text {INN }} \cap$ center $\Gamma \Gamma^{-1}=\Sigma_{\text {INN }} \cup \Sigma_{\text {INN }}^{-1}$.

(vi) An arbitrary totally ordered group $\Lambda$ is of the form $\Lambda=\Gamma \Gamma^{-1}$ where the subsemigroup $\Gamma=\{x \in \Lambda \mid x \leqslant e=1\}$ satisfies (a) and (b) above.

(vii) For any $x \in \Gamma, x \Gamma=\Gamma x=\{y \in \Gamma \mid y \leqslant x\}$; every one sided ideal of $\Gamma$ is two sided and also a convex interval.

For a proof of the previous as well as the remarks below see [11, 2.5-2.7].

1.7. REMARKS. 1. In the presence of (a), the hypothesis (b) above is equivalent to the following apparently weaker hypothesis $\left(b^{\prime}\right)$ :

$\left(b^{\prime}\right)$ the principal left ideals of $\Gamma$ form a chain and $\Gamma$ is right Ore. (See [11, 2.5(ii)].)

2. Conclusions (ii) and (iv) above can be equivalently reformulated as follows. For any totally ordered cancellative semigroup with an identity the following are equivalent:

(i) $\Gamma$ satisfies 1.6(a) and (b).

(ii) There exists a totally ordered group $\Lambda$ such that $\Gamma=\{g \in \Lambda \mid v g \leqslant e=1\}$.

In the next definition $\Gamma$ need not be commutative. It may be a group. For some applications of the next definition, see [8, 1.1, p. 288]. 
1.8. Definition. For any domain $R$, and for any totally ordered cancellative semigroup $\Gamma$, a valuation is a homomorphism of multiplicative semigroups $v$ : $R^{*} \equiv R \backslash\{0\} \rightarrow \Gamma$ satisfying the following for all $\alpha, \beta \in R^{*}$ :

(i) $v(\alpha \beta)=v \alpha(v \beta)$, and

(ii) $v(\alpha+\beta) \leqslant \operatorname{maximum}(v \alpha, v \beta) \equiv \max (v \alpha, v \beta)$.

Properties (i) and (ii) imply (iii):

(iii) $v \beta<v \alpha \Rightarrow v(\alpha+\beta)=v \alpha$.

1.9. For $R=K[\Gamma ; \phi]$ as in 1.1 , define a valuation $v$ and another semigroup homomorphism $\operatorname{deg}$ by $v, \operatorname{deg}: R^{*} \rightarrow \Gamma, \quad v(\alpha)=v \alpha=$ maximum supp $\alpha=$ $\max \operatorname{supp} \alpha$, and $\operatorname{deg} \alpha=\operatorname{minimum} \operatorname{supp} \alpha=\min \operatorname{supp} \alpha$ for $\alpha \in R^{*}$. In the same way $v$ and deg are defined for $K\left[\Gamma \Gamma^{-1} ; \theta\right]$ if $\Gamma$ is right Ore.

The next simple observation will later be frequently used automatically without explicit mention.

1.10. If $K[\Gamma ; \phi]$ is as in 1.4 and $0 \neq a \in K[\Gamma ; \phi]$, then

(i) $a \in K^{*} \Leftrightarrow \operatorname{deg} a=v a=e$.

(ii) If $\Gamma \leqslant e=1$, then $a \in K^{*} \Leftrightarrow \operatorname{deg} a=e$.

(iii) If $e \leqslant \Gamma$, then $a \in K^{*} \Leftrightarrow v a=e$.

1.11. Remarks. 1. For a symbol $0 \notin \Gamma$, form $\Gamma \cup\{0\}$ and define $0=0 x=x 0<x$ for all $x \in \Gamma$. Then $\Gamma \cup\{0\}$ is a totally ordered semigroup, and $v$ extends to a homomorphism $\bar{v}: R \rightarrow \Gamma \cup\{0\}$ of multiplicative semigroups by $\bar{v} 0=0$.

2. Suppose that $K$ is a totally ordered division ring, and that for every $x \in \Gamma, x \phi$ is an order preserving endomorphism of $K$, i.e. if $K^{+}=\{k \mid 0<k \in K\}$, then $K^{+}(x \phi)$ $\subseteq K^{+}$. Then the ring $R=K[\Gamma ; \phi]$ becomes a totally ordered ring $(0 \leqslant \alpha, 0 \leqslant \beta \in$ $R \Rightarrow 0 \leqslant \alpha+\beta$ and $0 \leqslant \alpha \beta \in R$ ), where an element $\alpha \in R^{*}$ is defined to be positive $0<\alpha$ if and only if $0<\alpha(v \alpha)$. In this case both $v$ and $\bar{v}$ are order preserving homomorphisms of totally ordered semigroups $\bar{v}: R^{+}=\{r \mid 0 \leqslant r \in R\} \rightarrow \Gamma \cup\{0\}$ and $v: R^{+*} \rightarrow \Gamma$.

3. The degree map deg satisfies 1.8(i)-(iii) with all inequalities reversed and with "max." replaced by "min.".

4. Although historically the degree map would be called a valuation, it seems more natural to define a valuation as in 1.8 for the following two reasons. (i) If $K$ is totally ordered, then in 2 above deg: $R^{+*} \rightarrow \Gamma$ is an order reversing semigroup map. (ii) Let $\Gamma \cup\{\infty\}$ be the semigroup defined by $x<\infty x=x \infty=\infty$ for all $x \in \Gamma$. Set $\operatorname{deg} 0=\infty$. Then not only is deg: $R^{+} \rightarrow \Gamma \cup\{\infty\}$ order reversing, but moreover $\Gamma \cup\{\infty\} \nsubseteq R=K[\Gamma ; \phi]$. However, for $v, \Gamma \cup\{0\} \subset K[\Gamma ; \phi]$.

2. Invariant elements. Since invariant elements of a ring generate principal ideals, the first step in the study of the ideal structure of a skew semigroup ring will be to look at invariant elements of the ring and the semigroup.

2.1. Lemma. For $\phi: \Omega \rightarrow$ End $K$ as above, and for any domain $R$, let $\Omega_{\mathrm{INN}}, \Omega_{\mathrm{IV}}$ and $R_{\mathrm{IV}}$ be the multiplicative semigroups defined previously. Let $x, y \in \Omega$ and $s, \gamma \in R^{*}=$ $R \backslash\{0\}$.

(i) If any two of $s, \gamma, s \gamma$ belong to $R_{\mathrm{IV}}$, then so does the third.

(ii) If any two of $x, y, x y$ belong to $\Omega_{\mathrm{INN}}\left(\right.$ or $\left.\Omega_{\mathrm{IV}}\right)$ then so also does the third. 
Proof. (i) If $s, s \gamma \in R_{\mathrm{IV}}$, then cancellation of $s$ in $s \gamma R=R s \gamma=s R \gamma$ gives $\gamma R=R \gamma$. Similarly when $\gamma, s \gamma \in R_{\mathrm{IV}}$, then $R s \gamma=s \gamma R=s R \gamma$, and again $R s=s R$ after the $\gamma$ is cancelled. The rest is clear.

2.2. For $R=K[\Omega ; \phi]$ as in 1.1 with $\Omega$ a cancellative semigroup and $\Gamma_{\mathrm{IV}}, R_{\mathrm{IV}}$ as in 1.3 the following hold for any $s \in \Omega$.

(i) $s R=R s \Rightarrow s \Omega=\Omega s$ and $s \phi \in$ Aut $K$.

(ii) If $s \phi \in$ Aut $K$, then $s R=R s \Leftrightarrow s \Omega=\Omega s$.

(iii) Hence $R_{\mathrm{IV}} \cap \Omega=\Omega_{\mathrm{IV}} \cap\{x \in \Omega \mid x \phi \in$ Aut $K\}$.

Proof. In general, for any $\gamma \in R$, supp $s \gamma=s(\operatorname{supp} \gamma)$, supp $\gamma s=(\operatorname{supp} \gamma) s$, and hence $|\operatorname{supp} s \gamma|=|\operatorname{supp} \gamma s|=|\operatorname{supp} \gamma|$. Also, $s R \cap \Gamma=s \Gamma$ and $R s \cap \Gamma=\Gamma s$. (i) For any $c \in K, s c=k s \in R s$ for some $k \in R$. It follows from $\{s\}=\operatorname{supp} k s=$ $(\operatorname{supp} k) s$ that $\operatorname{supp} k=\{e\}$ and $k \in K$. Thus $c=k(s \phi)$, and $s \phi$ is onto. The rest is clear.

In the next lemma below it is not assumed that $\Gamma \leqslant e=1$. Among other things it shows that in a skew semigroup ring $K[\Gamma ; \phi]_{\mathrm{IV}}$ and $\Gamma_{\mathrm{INN}}$ are not completely independent of one another.

2.3. LemMA. For $K[\Gamma ; \phi]$ as in 1.4 , assume that $\gamma K[\Gamma ; \phi]=K[\Gamma ; \phi] \gamma$ for some $0 \neq \gamma=\sum z \gamma(z) \in K[\Gamma ; \phi]$ with $y, e \in \operatorname{supp} \gamma$. Then for any element $k \in K^{*}$

(i) $k^{y}=\gamma(y) \gamma(e)^{-1} k \gamma(e) \gamma(y)^{-1}$.

(ii) If $\gamma(e) \in$ center $K$, then $k \gamma=\gamma k$.

Proof. (i) By hypothesis, $k \gamma=\gamma c$ for some $0 \neq c \in K[\Gamma ; \phi]$. Since $v k=\operatorname{deg} k=$ $e$, it follows that $\operatorname{deg}(k \gamma)=\operatorname{deg} \gamma=\operatorname{deg} \gamma(\operatorname{deg} c)$, and hence that $\operatorname{deg} c=e$; similarly $v(k \gamma)=v \gamma=v \gamma(v c)$, and $v c=e$. Thus also $c \in K^{*}$. It now follows from $k \gamma=\gamma c$ that for any $z \in \operatorname{supp} \gamma$

$$
\begin{gathered}
k^{z} \gamma(z)=\gamma(z) c, \\
c=\gamma(y)^{-1} k^{y} \gamma(y)=\cdots=\gamma(z)^{-1} k^{z} \gamma(z)=\cdots=\gamma(e)^{-1} k \gamma(e), \\
k^{y}=\gamma(y) \gamma(e)^{-1} k \gamma(e) \gamma(y)^{-1} .
\end{gathered}
$$

(ii) Evaluation of $k \gamma=\gamma c$ at $e$ proves (ii).

2.4. Proposition. For $R=K[\Gamma ; \phi]$ as in 1.4 with $\Gamma \leqslant e=1$, suppose that $0 \neq J \triangleleft$ $R$ is a biprincipal (1.1) ideal. Then there is an $\alpha \in R^{*}$ such that

(i) $J=\alpha R=R \alpha$.

(ii) For any $z \in \operatorname{supp} \alpha, z \phi \in$ Aut $K$.

(iii) If $s=v \alpha$ and $m=\operatorname{deg} \alpha$, then $s, m \in \Gamma_{\mathrm{IV}}$ and $s, m \in R_{\mathrm{IV}}$.

(iv) If $J=\bar{\alpha} R$ for some $\bar{\alpha} \in R$, then $\bar{\alpha}=\alpha k$ for some $k \in K$.

Proof. (i) If $J=\alpha R=R \beta \triangleleft R$ for $\alpha, \beta \in R$, then $\alpha=a \beta \in R \beta$ and $\beta=\alpha b \in$ $\alpha R$ for some $a, b \in K^{*}$. Thus $\alpha=a \alpha b$. Since $\operatorname{deg} \alpha=\operatorname{deg} a(\operatorname{deg} \alpha) \operatorname{deg} b$ it follows from $\Gamma \leqslant e$ that $\operatorname{deg} a=\operatorname{deg} b=e$. Thus $a, b \in K^{*}$. Consequently $J=R \beta=R a \beta$ $=R \alpha$. So $J=\alpha R=R \alpha$.

(ii) It will be shown that for any $c \in K^{*}$, there is a $k \in K^{*}$ such that $k(z \phi)=c$. Set $d=\alpha(z)^{-1} c \alpha(z)$. Then $\alpha d=k \alpha$ for some $k \in R$. Since $\operatorname{deg}\left(K^{*}\right)=\{e\}$, it 
follows that $\operatorname{deg} \alpha=(\operatorname{deg} k)(\operatorname{deg} \alpha), \operatorname{deg} k=e$, and hence that also $k \in K$. Thus $z \alpha(z) d=k z \alpha(z)=z k^{z} \alpha(z)$, or

$$
k^{z}=\alpha(z) d \alpha(z)^{-1}=c .
$$

(iii) For any $y \in \Gamma, \alpha y=\delta \alpha$ for some $\delta \in R$. Then

$$
v(\alpha y)=s y=(v \delta) s \in \Gamma s, \quad \operatorname{deg}(\alpha y)=m y=(\operatorname{deg} \delta) m \in \Gamma m .
$$

Hence $s \Gamma \subseteq \Gamma s$ and $m \Gamma \subseteq \Gamma m$. By symmetry of hypotheses, $s \Gamma=\Gamma s$ and $m \Gamma=\Gamma m$. Now 2.2(iii) together with 2.4(i) show that also $s, m \in R_{\mathrm{IV}}$.

(iv) If $\bar{\alpha} R=\alpha R$ is any principal right ideal, then $\alpha=\bar{\alpha} c, \bar{\alpha}=\alpha k$, and $\bar{\alpha}=\bar{\alpha} c k$ for some $c, k \in R^{*}$. Thus $\operatorname{deg} \bar{\alpha}=\operatorname{deg} \bar{\alpha}(\operatorname{deg} c) \operatorname{deg} k \in \Gamma \leqslant e$ implies that $\operatorname{deg} c=$ $\operatorname{deg} k=1$, and $k \in K^{*}$.

2.5. COROllary 1. With $R=K[\Gamma ; \phi]$ as in 1.4 assume in addition that

(a) $\Gamma \leqslant e=1$, and that $(b) \Gamma$ is commutative.

Suppose that $\beta R=R \beta$ for some $\beta \in R$ with $0 \neq \beta(e) \in K$. Set $\gamma=\beta \beta(e)^{-1}=$ $\sum z \gamma(z)$ and let $z \in \operatorname{supp} \gamma$ be arbitrary. Then

(i) $\gamma R=R \gamma, \gamma \in$ center $R$.

(ii) For any $k \in K, k^{z}=\gamma(z) k \gamma(z)^{-1}, \gamma(z) \in K_{\phi}$.

Proof. (i) Since $\beta \beta(e)^{-1} R=\beta R$ and since $R \beta \beta(e)^{-1}=\beta R \beta(e)^{-1}=\beta R$, also $\gamma R=R \gamma$. For any $x \in \Gamma, x \gamma=\gamma \delta$ for some $\delta \in R$. It follows from the commutativity of $\Gamma$ and from $x \operatorname{deg} \gamma=\operatorname{deg} \gamma(\operatorname{deg} \delta)$ and $x=v(x \gamma)=v \gamma(v \delta)=v \delta$ that $\operatorname{deg} \delta$ $=v \delta=x$, and hence that $\delta=x c$ for some $c \in K$. Evaluation of $x \gamma=\gamma x c$ at $e$ gives $x=x \gamma(e)=\gamma(e) x c=x c$, or $c=1$. Next, for any $k \in K^{*}, k \gamma=\gamma \rho$ with $\rho \in R^{*}$. But then $\operatorname{deg} \gamma=(\operatorname{deg} \gamma) \operatorname{deg} \rho$ and hypothesis (a) imply that $\rho \in K^{*}$. Thus $k=$ $k \gamma(e)=\gamma(e) \rho=\rho$. Hence $\gamma \in$ center $R$.

(ii) It follows as a very special case of $[11,2.2]$ that any central element satisfies these conditions.

2.6. CoRollaRy 2. If in the last proposition $J=\alpha R=R \beta \triangleleft R=K[\Gamma ; \phi]$ for any $\alpha, \beta \in R^{*}$ whatever, then $J=\alpha R=R \alpha, J=R \beta=\beta R$, and $\beta=\alpha k$ for some $k \in K^{*}$.

2.7. REMARK. If in the last proposition $y \neq z \in \operatorname{supp} \alpha$, than it follows from the proof of 2.3 and 2.4(ii) that $(z \phi)^{-1}(y \phi)$ is an inner automorphism of $K$. Thus in the quotient group of Aut $K$ modulo the inner automorphisms, the set $\operatorname{supp} \alpha$ is contained in a single coset.

3. Ideals. The end objective of this section is to determine completely an arbitrary biprincipal ideal in a class of skew semigroup and group rings as well as to construct some simple rings. In order to avoid assuming restrictive assumptions on $\Gamma$ which are actually never used in the proofs, and whose only purpose is to guarantee that the skew semigroup ring is both a prid and a plid, most of the results in this section are formulated for biprincipal ideals.

It follows from the symmetry of the conclusions that the next proposition holds verbatum if instead of (b) it is assumed that the principal left ideals of $\Gamma$ form a chain. 
3.1. Proposition. For the skew semigroup ring $R=K[\Gamma ; \phi]$ as in 1.4 with the additional assumptions that (a) $\Gamma \leqslant e=1$, and that (b) the principal right ideals of $\Gamma$ are linearly ordered, suppose that $J \triangleleft R$ is a nonzero biprincipal ideal, i.e. $0 \neq J=\alpha R$ $=R \beta$ for some $\alpha, \beta \in R^{*}$. Then $J$ is of the form

(i) $J=s \gamma R=R s \gamma ; s \in \Gamma, \gamma \in R$; for any $z \in \operatorname{supp} \gamma, s \phi, z \phi \in$ Aut $K$; $\gamma(e)=1$.

(ii) $s \Gamma=\Gamma s, s R=R s, \gamma R=R \gamma$.

(iii) $(\operatorname{deg} \gamma) \Gamma=\Gamma(\operatorname{deg} \gamma),(\operatorname{deg} \gamma) R=(\operatorname{deg} \gamma) R$.

(iv) If $J=\bar{s} \bar{\gamma} R$ with $\bar{s} \in \Gamma, \bar{\gamma} \in R$, and $\bar{\gamma}(e)=1$, then $\bar{s}=s$ and $\delta=\gamma$. (The analogue also holds on the right side if $J=R \bar{s} \bar{\gamma}$.)

Proof. (i) By 2.4(i), $J=\alpha R=R \alpha$. Set $s=v \alpha$. For $x \in \operatorname{supp} \alpha \leqslant s$ by (b) and 1.6(i), $x=s z$ with $z \in \Gamma$. Thus $\alpha=s \gamma$ where $\gamma=\sum z \gamma(z) \in R, \gamma(z)=\alpha(s z) \in K$, with $v(\gamma)=e$, and $0 \neq \gamma(e)=\alpha(s) \in K^{*}$. Thus $s \gamma R=R s \gamma$. Since $s \gamma \gamma(e)^{-1} R=$ $s \gamma R$, and since $R s \gamma \gamma(e)^{-1}=s \gamma R \gamma(e)^{-1}=s \gamma R$, it follows that $J=s \gamma \gamma(e)^{-1} R=$ $R s \gamma \gamma(e)^{-1}$. Hence without loss of generality - by replacing $\gamma$ with $\gamma \gamma(e)^{-1}$-it may be assumed that $\gamma(e)=1$.

(ii) By 2.4(iii), $s=v \alpha \in R_{\mathrm{IV}} \cap \Gamma_{\mathrm{IV}}$. Since $s \gamma \in R_{\mathrm{IV}}$ and $s \in R_{\mathrm{IV}}, 2.1$ (i) shows that also $\gamma \in R_{\text {IV }}$. (iii) But then also $\operatorname{deg} \gamma \in R_{\text {IV }} \cap \Gamma_{\text {IV }}$ by 2.4(iii). (iv) Lastly $\bar{s} \bar{\gamma}=s \gamma k$ for some $k \in K^{*}$ by $2.4(\mathrm{iv})$. Then $\bar{s}=v(\bar{s} \bar{\gamma})=v(s \gamma k)=s, \bar{\gamma}=\gamma k$, and $k=1$ because $1=\bar{\gamma}(e)=\gamma(e) k=k$.

By using a different method of proof the next theorem is able to slightly extend $[11,3.5]$.

3.2. TheOREM I. For a totally ordered cancellative semigroup $\Gamma$ with (a) $\Gamma \leqslant e=1$ whose (b) principal right ideals form a chain, as before let $\phi: \Gamma \rightarrow$ End $K$ for a skew field $K$, and let $K[\Gamma ; \phi]$ be the resulting skew semigroup ring. As previously $C_{\phi} \subseteq K$ is the subfield of the center of $K$ which is left elementwise fixed by $\Gamma$. Suppose that $J \triangleleft K[\Gamma ; \phi]$ is a biprincipal ideal, i.e. a principal right and also a principal left ideal. If $\Gamma$ has the property (N.I.)

(N.I.) for any $e \neq y \in \Gamma, y \phi$ is NOT inner, then

(i) $J=s K[\Gamma ; \phi]=K[\Gamma ; \phi]$ s for some unique $s \in \Gamma$; furthermore $s \Gamma=\Gamma s$, and $s \phi$ is an automorphism of $K$.

(ii) center $K[\Gamma ; \phi]=C_{\phi}$.

Proof. (i) Set $R=K[\Gamma ; \phi]$. By 3.1, $J=s \gamma R=R s \gamma$ where $\gamma \in R, s \in \Gamma$, with $\gamma R=R \gamma$, and $s \Gamma=\Gamma$ s. Let $y=\min \operatorname{supp} \gamma \leqslant e$. By Lemma 2.3, for all $k \in K^{*}$, $k^{y}=\gamma(y) \gamma(e)^{-1} k \gamma(e) \gamma(y)^{-1}$. By hypothesis (N.I.), $y=e$, min supp $\gamma=v \gamma$, and $\gamma=\gamma(e) \in K^{*}$. But then $J=s R=R s$. The rest of (i) follows from 3.1.

(ii) If $0 \neq \alpha \in$ center $R$, set $y=\operatorname{deg} \alpha \in \Gamma$. For any $k \in K$, it follows from $k \alpha=\alpha k$ that

$$
k y \alpha(y)=y k^{y} \alpha(y)=y \alpha(y) k \quad \text { and } \quad k^{y}=\alpha(y) k \alpha(y)^{-1} .
$$

The property (N.I.) requires that $y=e$ and hence that $\alpha \in K$. But $K_{\phi} \cap$ center $R=$ $C_{\phi}$. Thus center $R=C_{\phi}$. 
3.3. THEOREM II. Suppose that $\Gamma$ is a totally ordered cancellative semigroup and $\theta$ : $\Gamma \rightarrow$ Aut $K$ is a semigroup homomorphism. As before, $C_{\theta} \subseteq K$ is the subfield of the center of $K$ left elementwise fixed by $\Gamma$. Assume that

(a) $\Gamma \leqslant e=1$.

(b) The principal right and principal left ideals of $\Gamma$ form chains.

Form the skew semigroup rings $R \equiv K[\Gamma ; \theta] \subset S \equiv K\left[\Gamma \Gamma^{-1} ; \theta\right]$ as in 1.4. Then any biprincipal ideal $0 \neq I \triangleleft S$ is of the form

(i) $I=\gamma S=S \gamma, \gamma \in R, \gamma R=R \gamma, \gamma(e)=1$.

(ii) If $I=\bar{\gamma} S$ for $\bar{\gamma} \in R$ with $\bar{\gamma}(e)=1$, then $\bar{\gamma}=\gamma$. (The same holds for $I=S \bar{\gamma}$.)

(iii) If $\Gamma$ is commutative then $\gamma \in$ center $R \subset$ center $S$ in (i).

Finally, if in addition to the hypotheses (a) and (b), also

(N.I.) for any $e \neq y \in \Gamma, y \theta$ is not inner, then

(iv) $S$ is a simple ring, and

(v) center $S=$ center $R=C_{\theta}$.

Proof. (i) Let $I=\xi S=S \eta$ for some $\xi, \eta \in S^{*}$. It is known [11, 3.3(i)] that any elements such as $\xi, \eta \in S^{*}$ can be written uniquely in the form $\xi=\alpha a x, \eta=y b \beta$, where $\alpha, \beta \in R^{*}$ with $\alpha(e)=\beta(e)=1, a, b \in K^{*}$; and $x, y \in \Gamma \Gamma^{-1}=\Gamma \cup \Gamma^{-1}$. Hence $I=\alpha S=S \beta$. Set $J=I \cap R \triangleleft R$. Since $\alpha(e)=1, \alpha S \cap R=\alpha R$. Thus $J=\alpha R=R \beta$. By 3.1(i) (with $s=e$ ), $J$ is of the form $J=\gamma R=R \gamma$, where $\gamma \in R^{*}$, $\gamma R=R \gamma$, and $\gamma(e)=1$. (The argument used in the proof of 3.1 actually showed that $\gamma=\alpha$.) It is a consequence of $I=\alpha S=\alpha R S$ that $I=J S=S J$, and hence that $I=\gamma S=S \gamma$.

(ii) If $\gamma S=\bar{\gamma} S$ is any right ideal of $S$ where $\gamma, \bar{\gamma} \in R$ with $\gamma(e)=\bar{\gamma}(e)=1$, then $\gamma R=\gamma S \cap R=\bar{\gamma} R$. By 3.1(iv), $\gamma=\bar{\gamma}$.

(iii) Although the proof of (i) that $J=\gamma S=S \gamma$ required the hypothesis (b) once given an element $\gamma \in R$ as in (i), merely hypothesis (a) and the commutativity of $\Gamma$ are sufficient to guarantee conclusion (iii) by 2.5(i).

(iv) Any $0 \neq I \triangleleft S$ is of the form $I=\gamma S=S \gamma$ with $\gamma \in R$ and $\gamma(e)=1$ by 3.3(i). Use of the hypothesis (N.I.) and 2.3 shows that supp $\gamma=\{e\}$. Hence $\gamma=\gamma(e)=1$.

(v) By [11, 3.7(ii)] hypotheses (a) and (b) alone are already sufficient to prove that center $S=$ center $R=C_{\theta}$.

It will be shown in 3.4 and 3.5 below how the simple ring $S$ given by the previous theorem can be embedded in a division ring.

3.4. Construction. Suppose that $K$ is a skew field, $\Lambda$ a totally ordered group, and $\theta$ : $\Lambda \rightarrow$ Aut $K$ a group homomorphism. Let $K((\Lambda ; \theta))$ denote the abelian group of all functions $\alpha, \beta: \Lambda \rightarrow K$ whose supports satisfy the ascending chain condition (A.C.C.). The product $\alpha \beta$ of $\alpha$ and $\beta$ is defined exactly as in 1.1. Then it is known and is not difficult to show that $K((\Lambda ; \theta)$ ) is a division ring. (See [6, p. 366, Lemma 1.5; 7, p. $27,1.2(\mathrm{a})$ and (b), or 9, p. 974, 2.16].)

Conclusion (ii) below is proved in [11, 3.7(ii)].

3.5. Corollary 1 to Theorem II. With the notation and under the hypotheses (a) and (b) of the previous theorem, the rings $R \subset S$ can be embedded in the division $K\left(\left(\Gamma \Gamma^{-1} ; \theta\right)\right)$ : 
(i) $R \subset S \subset K\left(\left(\Gamma \Gamma^{-1} ; \theta\right)\right)$.

If (a), (b), and (N.I.) hold, then

(ii) center $R=$ center $S=$ center $K\left(\left(\Gamma \Gamma^{-1} ; \theta\right)\right)=C_{\theta}$.

Although in view of $1.6(\mathrm{vi})$ the next corollary is of course logically equivalent to the next theorem, nevertheless it is useful because in practice one rarely starts with $\Gamma$ satisfying the lengthy hypotheses of the last theorem, while skew group rings occur naturally. That is, a ring of the below form $K[\Lambda ; \theta]$ can be concisely defined and thus seems to be more familiar.

3.6. Corollary 2 to Theorem II. Starting from any totally ordered group $\Lambda, a$ skew field $K$, and any group homomorphism $\theta: \Lambda \rightarrow$ Aut $K$, now define $\Gamma$ as the semigroup $\Gamma=\{g \in \Lambda \mid g \leqslant e=1\}$ and then form the rings $R \equiv K[\Gamma ; \theta] \subset S \equiv$ $K[\Lambda ; \theta]$. Then $\Gamma$ satisfies the hypotheses 3.3(a) and (b), and hence $R \subset S$ satisfy all the conclusions of Theorem II. In particular, if $y \theta$ is NOT an inner automorphism of $K$ for any $e \neq y \in \Gamma$, then $K[\Lambda ; \theta]$ is a simple ring.

4. Applications and examples. Below is a reformulation of a result which appears in $[15$, p. 38]. One difference is that unlike the situation in [15], below $\gamma$ and $\alpha$ are central elements; thus the connection between ideals and central elements is brought out. Also uniqueness is added. For a detailed concrete description of the central elements $\gamma$ and $\alpha$ below see [11, 3.1-3.2]. This corollary illustrates how all the technical hypotheses of the last two theorems simply disappear because they are automatically satisfied.

4.1. For an automorphism $\theta: K \rightarrow K$ of a skew field $K$, let $C_{\theta} \subseteq K$ be the subfield $C_{\theta}=\{c \in$ center $K \mid c \theta=c\}$, and let $\Gamma=\left\{1>x>x^{2}>\cdots\right\}$ be the infinite cyclic semigroup. Any element $x^{n} \in \Gamma$ induces an automorphism of $K \rightarrow K$ by $k \rightarrow k \theta^{n}$. Although strictly speaking $\theta$ should map $\Gamma \rightarrow$ Aut $K$, it is a convention of long standing to denote the skew semigroup rings for $\Gamma \subset \Gamma \Gamma^{-1}$ in this case by $K[x ; \theta] \subset$ $K\left[x, x^{-1} ; \theta\right]$ respectively. For any polynomials $\gamma$ or $\alpha$ of $K[x ; \theta], \gamma(0)$ and $\alpha(0) \in K$ denote their constant terms.

4.2. Corollary to Theorems I AND II. For the skew polynomial rings $R \equiv$ $K[x ; \theta] \subset K\left[x, x^{-1} ; \theta\right] \equiv S$ if $0 \neq J \triangleleft R$ and $0 \neq I \triangleleft S$ are any ideals, then

(i) $J=x^{k} \gamma R=R x^{k} \gamma ; k=0,1,2, \ldots ; \gamma \in$ center $R$.

(ii) If $J=x^{m} \bar{\gamma} R$ where $\bar{\gamma} \in R$ with $\bar{\gamma}(0)=1$, and if $\gamma(0)=1$, then $m=k$ and $\bar{\gamma}=\gamma$. (The same holds on the right.)

(iii) $I=\alpha S=S \alpha, \alpha \in$ center $R \subset$ center $S$.

(iv) If $I=\bar{\alpha} S$, where $\bar{\alpha} \in S$ with $\bar{\alpha}(0)=1$, and if $\alpha(0)=1$, then $\bar{\alpha}=\alpha$. (The same holds if $\bar{\alpha}$ is on the right.)

(N.I.) If for any $1 \leqslant n, \theta^{n}$ is not inner, then

(v) $J=x^{k} R=R x^{k}$ for some unique $k=0,1,2, \ldots$

(vi) $S$ is simple.

(vii) center $R=$ center $S=C_{\theta}$.

The next example shows that there exist semigroups satisfying the hypotheses of 3.1 but not those of 3.3. 
4.3. ExAmple. Let $Q$ denote the rationals with the usual natural order. Then $Q \times Q$ becomes a totally ordered group under the following operations:

$$
\begin{gathered}
(n, i)(m, j)=(n+m, 2 i m+j), \quad n, i, m, j \in Q ; \\
(n, i)<(m, j) \quad \text { if either } n>m, \text { or } n=m \text { and } i>j .
\end{gathered}
$$

Let $Z=0, \pm 1, \pm 2, \ldots, Z^{+}=0,1,2, \ldots$, and $\Gamma \subset Q \times Q$ be the nonconvex subsemigroup $\Gamma=\left\{(n, i) \mid 1 \leqslant n \in Z^{+}, i \in Z\right\} \cup\{0\} \times Z^{+}$. If $(n, i)<(m, j) \in \Gamma$, then $(n, i)=(m, j)(n-m, i-2 j(n-m)) \in(m, j) \Gamma$. Thus $\Gamma \leqslant e=(0,0)$ and the principal right ideals of $\Gamma$ form a chain. Since $\Gamma(1,0) \cap \Gamma(1,1)=\varnothing$, the principal left ideals of $\Gamma$ are not linearly ordered.

The next counterexample shows that $\gamma \in K[\Gamma ; \phi], x \in \Gamma$, and $\gamma x=\delta \gamma$ with $2 \leqslant|\operatorname{supp} \delta|$ is possible.

4.4. Counterexample. Let $\Gamma$ be as in the previous example, $x=(1,0)$, and $t=(0,1)$. Then in the ordinary semigroup ring $K[\Gamma]$ over any field $K,(t-1) x=$ $(x t+x)(t-1)$.

The method of constructing a simple ring in the next example can be generalized.

4.5. ExAmple. Let $Q \subset Q(\sigma, \tau)=K$ be a transcendental extension field of the rationals $Q$ of transcendence degree two obtained by adjoining two transcendental independent indeterminates $\sigma$ and $\tau$. For $Z^{+}=\{0,1,2, \ldots\} \subset Z=\{0, \pm 1, \pm 2, \ldots\}$ as before, let $Z \times Z$ be the commutative totally ordered group $(i, j)(m, n)=(i+$ $m, j+n),(i, j)<(m, n)$ if either $i>m$, or $i=m$ but $j>n$. Let $\Gamma$ be the convex subsemigroup $\Gamma=\{g \in Z \times Z \mid g \leqslant e=(0,0)\}=Z^{+} \times Z^{+}$. In order to define a group homomorphism $\theta: Z \times Z \rightarrow$ Aut $K$, it suffices to define $\theta$ on the generators $(1,0)$ and $(0,1)$ of the free abelian group $Z \times Z$ :

$$
\begin{array}{llrl}
\sigma[(1,0) \theta] & =\sigma+1, & \tau[(1,0) \theta] & =\tau, \\
\sigma[(0,1) \theta] & =\sigma, & \tau[(0,1) \theta] & =\tau+1 .
\end{array}
$$

The right Ore quotient group $\Gamma \Gamma^{-1}$ of $\Gamma$ is $\Gamma \Gamma^{-1}=Z \times Z$. If $R \subset S$ are the resulting rings $R=K\left[Z^{+} \times Z^{+} ; \theta\right] \subset S=K[Z \times Z ; \theta]$, then $S$ is simple by Theorem II, while every biprincipal ideal of $R$ is uniquely of the form $(i, j) R=R(i, j)$ for some $(i, j) \in Z^{+} \times Z^{+}$by Theorem $\mathrm{I}$.

Set $x=(1,0)$ and $y=(0,1)$. The subring $T=K\left[Z^{+} \times\{0\} ; \theta\right] \subset R$ has the structure of a skew polynomial ring $T=K[x ; x \theta]$. Since $(x \theta) y \theta=y \theta(x \theta)$ commute, the automorphism $y \theta: K \rightarrow K$ extends to a ring automorphism $y \theta: T \rightarrow T$ by defining $\left(x^{n} k\right) y \theta=x^{n} k^{y}$. Thus $R$ may be regarded as a skew polynomial ring $R=T[y ; y \theta]$ in the indeterminate $y$ with coefficients in $T$. This was possible only because $\Gamma \theta$ was abelian. Although this easy example could have been handled by the already familiar skew polynomial rings, nevertheless the more general skew semigroup ring description developed here seems less cumbersome.

\section{REFERENCES}

1. P. M. Cohn, Free rings and their relations, London Math. Soc. Monographs, Academic Press, London, 1971.

2. Skew field constructions, Cambridge Univ. Press, London, 1977.

3. P. Conrad, Ordered semigroups, Nagoya Math. J. 16 (1960), 51-64.

4. P. Conrad and J. Dauns, An embedding theorem for lattice ordered fields, Pacific J. Math. 30 (1969), $385-398$ 
5. P. Conrad and P. McCarthy, The structure of f-algebras, Math. Nachr. 58 (1973), 169-191.

6. J. Dauns, Integral domains that are not embeddable in division rings, Pacific J. Math. 34 (1970), $27-31$.

7. Semigroup power series rings, Pacific J. Math. 34 (1970), 365-369.

8. Embeddings in division rings, Trans. Amer. Math. Soc. 150 (1970), 287-299.

9. Ordered domains, Symposia Mathematicae, vol. 21, Academic Press, London, 1977, pp. 565-587.

10. Noncyclic division rings, Math. Z. 169 (1979), 195-204.

11. Generalized semigroup rings, Algebra Carbondale 1980, Lecture Notes in Math., vol. 848 . Springer, New York, 1981.

12. L. Fuchs, Partially ordered algebraic systems, Pergamon Press, New York; Addison-Wesley, Reading, Mass., 1963.

13. Teilweise geordnete algebraische Strukturen, Akad. Kiado, Budapest, 1966.

14. C. Holland, A totally ordered integral domain with a convex left ideal which is not an ideal, Proc. Amer. Math. Soc. 11 (1960), 703.

15. N. Jacobson, The theory of rings, Math. Surveys, no. 2, Amer. Math. Soc., Providence, R.I., 1943.

16. A. V. Jategaonkar, Left principal ideal domains, J. Algebra 8 (1968), 148-155.

17. A. I. Malcev, On embedding of group algebra in a division ring, Dokl. Akad. Nauk SSSR 60 (1948). 1499-1501.

18. S. Montgomery and D. S. Passman, Crossed products over prime rings, Israel J. Math. 31 (1978), $224-256$.

19. D. S. Passman, Algebraic structure of group rings, Wiley-Interscience, New York, 1977.

20. W. Powell, Projectives in a class of lattice ordered modules, Algebra Universalis (to appear).

21. M. Satyanarayana, Principal right ideal semigroups, J. London Math. Soc. 3 (1971), 549-553.

22. S. Stcinberg, An embedding theorem for commutative lattice-ordered domains, Proc. Amer. Math. Soc. 31 (1972), 409-416.

Department of Mathematics, Tulane University, New Orleans, Louisiana 70118 